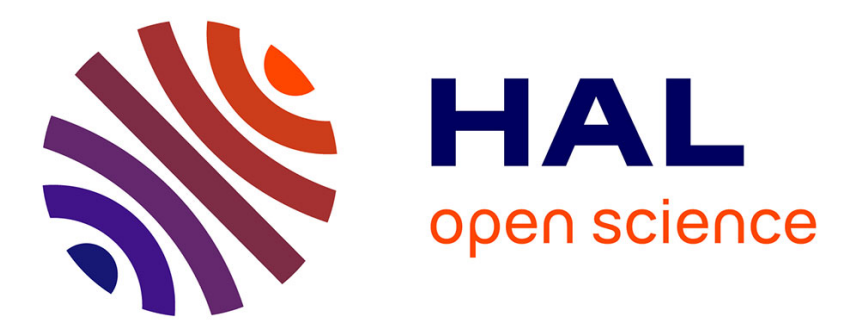

\title{
Noncollinear Ordering of the Orbital Magnetic Moments in Magnetite
}

H. Elnaggar, Ph Sainctavit, A. Juhin, S. Lafuerza, F. Wilhelm, A. Rogalev, M.-A. Arrio, Ch. Brouder, M. van Der Linden, Z. Kakol, et al.

\section{- To cite this version:}

H. Elnaggar, Ph Sainctavit, A. Juhin, S. Lafuerza, F. Wilhelm, et al.. Noncollinear Ordering of the Orbital Magnetic Moments in Magnetite. Physical Review Letters, 2019, 123 (20), 10.1103/PhysRevLett.123.207201 . hal-03004836

\section{HAL Id: hal-03004836 https://hal.science/hal-03004836}

Submitted on 13 Nov 2020

HAL is a multi-disciplinary open access archive for the deposit and dissemination of scientific research documents, whether they are published or not. The documents may come from teaching and research institutions in France or abroad, or from public or private research centers.
L'archive ouverte pluridisciplinaire HAL, est destinée au dépôt et à la diffusion de documents scientifiques de niveau recherche, publiés ou non, émanant des établissements d'enseignement et de recherche français ou étrangers, des laboratoires publics ou privés. 


\title{
Non-collinear ordering of the orbital magnetic moments in magnetite
}

\author{
H. Elnaggar, ${ }^{1, *}$ Ph. Sainctavit,${ }^{2}$ A. Juhin,${ }^{2}$ S. Lafuerza,${ }^{3}$ F. Wilhelm, ${ }^{3}$ A. Rogalev,${ }^{3}$ M.- A. Arrio,${ }^{2}$ Ch. Brouder, ${ }^{2}$ \\ M. van der Linden, ${ }^{1,3}$ Z. Kakol, ${ }^{4}$ M. Sikora, ${ }^{5}$ M. W. Haverkort,${ }^{6}$ P. Glatzel, ${ }^{3}$ and F. M. F. de Groot ${ }^{1, \dagger}$ \\ ${ }^{1}$ Debye Institute for Nanomaterials Science, Utrecht University, 3584 CA Utrecht, The Netherlands. \\ ${ }^{2}$ Institut de Minéralogie, de Physique des Matériaux et de Cosmochimie, CNRS, \\ Sorbonne Université, MNHN, UMR7590, 75252 Paris Cedex 05, France. \\ ${ }^{3}$ European Synchrotron Radiation Facility, CS40220, F-38043 Grenoble Cedex 9, France. \\ ${ }^{4}$ Faculty of Physics and Applied Computer Science, \\ AGH University of Science and Technology, Mickiewicza 30, 30-059 Krakow, Poland. \\ ${ }^{5}$ Academic Centre for Materials and Nanotechnology, \\ AGH University of Science and Technology, Mickiewicza 30, 30-059 Krakow, Poland. \\ ${ }^{6}$ Institut für Theoritiche Physik, Universität Heidelberg, Philosophenweg 19, 69120 Heidelberg, Germany.
} (Dated: November 13, 2020)

\begin{abstract}
The magnitude of the orbital magnetic moment [1-9] and its role as a trigger of the Verwey transition [10-17] in the prototypical Mott insulator, magnetite, remain contentious. Using $1 s 2 p$ resonant inelastic X-ray scattering angle distribution (RIXS-AD), we prove the existence of noncollinear orbital magnetic ordering and infer the presence of dynamical distortion creating a polaronic precursor for the metal to insulator transition. These conclusions are based on a subtle angular shift of the RIXS-AD spectral intensity as a function of the magnetic field orientation. Theoretical simulations show that these results are only consistent with non-collinear magnetic orbital ordering. To further support these claims we perform Fe $K$-edge X-ray magnetic circular dichroism (XMCD) in order to quantify the iron average orbital magnetic moment.
\end{abstract}

Magnetite $\left(\left[\mathrm{Fe}^{3+}\right]_{\mathrm{A}}\left[\mathrm{Fe}^{3+}, \mathrm{Fe}^{2+}\right]_{\mathrm{B}} \mathrm{O}_{4}\right)$ is the most abundant iron bearing mineral on Earth and it finds many applications in areas such as palaeomagnetism, medicine, data recording, and engineering [18]. Ever since Verwey's pioneering work [19], an immense amount of research has been dedicated to $\mathrm{Fe}_{3} \mathrm{O}_{4}$ in view of its importance as a reference for systems exhibiting the metal to insulator transition [11, 20-22]. In $\mathrm{Fe}_{3} \mathrm{O}_{4}$, the Verwey transition occurs at $T_{V} \sim 125 \mathrm{~K}$ and results in a spontaneous change of both, the lattice symmetry and the electric conductivity. Above $T_{V} \mathrm{Fe}_{3} \mathrm{O}_{4}$ has a cubic inverse spinel crystal structure containing two different $\mathrm{Fe}$ sites. $\mathrm{Fe}^{3+}$ ions reside in tetrahedral $\left(T_{d}\right)$ interstices (the A sites) while both $\mathrm{Fe}^{2+}$ and $\mathrm{Fe}^{3+}$ ions reside in octahedral $\left(O_{h}\right)$ interstices (the B sites). The A and B sublattices are antiferromagnetically coupled while the Fe ions in the same sublattice are ferromagnetically coupled (Fig. 1a).

In ferromagnets and ferrimagnets, the spin and orbital magnetic moments of the transition metal ions can be directly quantified by applying the sum rules on the $L_{2,3}$ XMCD signal [23]. In spite of the great success of sum rules, the experimental and analysis procedures were shown to be prone to huge uncertainties due to surface effects [1]. Orbital magnetic moments as small as $0.01 \mu_{\mathrm{B}}$ [2] and as large as $0.33 \mu_{\mathrm{B}}[3]$ were reported for $\mathrm{Fe}_{3} \mathrm{O}_{4}$. In addition, large orbital magnetic moment contributions that are of equal absolute values but with antiparallel coupling between the $\mathrm{Fe} \mathrm{A}$ and $\mathrm{B}$ sublattices were also suggested [4] (for a unit formula of $\mathrm{Fe}_{3} \mathrm{O}_{4}: F e_{B} \mu_{L}=$ $1 \mu_{\mathrm{B}}$ and $\left.F e_{A} \mu_{L}=-1 \mu_{\mathrm{B}}\right)$. A summary of the orbital and spin magnetic moments reported in literature using various techniques is shown in Fig. S1 and Refs. [24-28]. These large discrepancies regarding an essential quantity to many Mott insulators [29] ask for a new approach.

In this work we employed a combination of $\mathrm{Fe} K$-edge $\mathrm{XMCD}$ and $1 s 2 p$ RIXS-AD measurements to investigate the orbital magnetic moment of $\mathrm{Fe}$ in $\mathrm{Fe}_{3} \mathrm{O}_{4}$. Experiments at the Fe $K$-edge $(1 s \rightarrow 3 d+4 p$ excitations $)$ have a probing depth of few $\mu m$ and hence surface effects are negligible offering a valuable advantage over $L_{2,3}$-edge measurements [30-34]. We quantified the average orbital magnetic moment by performing $K$-edge XMCD. The accurately measured $1 s 2 p$ RIXS-AD signal was used to determine the average square orbital magnetic moment which complements the average quantity obtained from XMCD. Combining these two experiments, it is possible to deduce whether the orbital magnetic moments of the Fe ions are parallel, antiparallel or non-collinear.

Guided by theoretical calculations, we show that the only possibility to explain both of our experimental results is the existence of a non-collinear magnetic orbital ordering that can tilt the orbital magnetic moment as much as $82^{\circ}$ away from the spin magnetic moment. We propose a model for this non-collinear orbital magnetic ordering and infer the presence of a dynamical distortion related to the $X_{3}$ phonon mode in the high temperature phase. Our model imposes strong restrictions on the candidate mechanisms for the Verwey transition $[10,35]$.

We investigated highly stoichiometric (001), (110) and (111) $\mathrm{Fe}_{3} \mathrm{O}_{4}$ single crystals at room temperature. The average orbital magnetic moment projected along the magnetic field direction can be quantified by performing Fe $K$ pre-edge XMCD measurements [23]. Three main dichroic features can be seen at $E_{I}=7112.7 \mathrm{eV}, E_{I I}=7114 \mathrm{eV}$ and $E_{I I I}=7115.1 \mathrm{eV}$ with only significant angular dependence at the first feature (Fig. 1b). The co-existence 
of two $\mathrm{Fe}$ sites in $\mathrm{Fe}_{3} \mathrm{O}_{4}$ complicates the direct analysis of the XMCD signal and renders a full calculation important to assign spectral features to the specific Fe species.

Configuration interaction calculations taking into account i)- intra-atomic Coulomb interaction, ii)- crystal field, iii)- spin-orbit coupling, and iv)- exchange interaction were performed using the quantum many-body program Quanty [36-38]. Our theoretical simulations show that the feature at $E_{I}$ arises mainly from electric quadrupole transitions (i.e. $1 s \rightarrow 3 d$ excitations) at the formal $\mathrm{Fe}^{2+}$ ions while the features at $E_{I I}$ and $E_{I I I}$ arise mainly from electric dipole transitions (i.e. $1 s \rightarrow 3 d+4 p$ excitations) at the $\mathrm{Fe}^{3+} T_{d}$ ions due to onsite $3 d-4 p$ orbital mixing as discussed by in Refs. [39-41]. The quadrupolar signals from the $\mathrm{Fe}^{3+} \mathrm{A}$ and $\mathrm{B}$ sites nearly cancel out as shown in Fig. S8.

The experimental XMCD signal and its angular dependence can be best interpreted to arise from a partially quenched orbital magnetic moment at the formal $\mathrm{Fe}^{2+}$ ions. An excellent agreement between the calculation and the experiment is observed (compare spectra labelled Exp and Calc 1 in Fig. 1b). This partial quenching is a result of the octahedral symmetry accompanied by a small trigonal distortion $\left(D_{\sigma}=67 \pm 10 \mathrm{meV}\right)$. The Fe environment is not perfectly $O_{h}$ since the point group symmetry of the B site is rhombohedral $\left(D_{3 d} \equiv \overline{3} m\right)$. We found that the average orbital magnetic moment is $0.26 \pm 0.03 \mu_{\mathrm{B}}$ per unit formula of $\mathrm{Fe}_{3} \mathrm{O}_{4}$. It is important to note that feature $\mathrm{I}$ is theoretically predicted to be completely suppressed in the case that $\mu_{L}=0 \mu_{\mathrm{B}}$ (see Fig. 1b Calc 2) strongly supporting the presence of a finite orbital magnetic moment in bulk $\mathrm{Fe}_{3} \mathrm{O}_{4}$.

To investigate possible non-collinearity of the orbital magnetic moment, we performed comprehensive $1 s 2 p$ RIXS-AD measurements. This complements the average projected result obtained from XMCD. The RIXS$\mathrm{AD}$ was measured by rotating the sample about the incident wave-vector direction $\left(\boldsymbol{k}_{\boldsymbol{i n}}\right)$ aligned with the [110] direction (refer to Fig. 2a). The RIXS-AD signal arises from both the crystal field (see Fig. S14) and from the magnetization of the absorbing atom (see Fig. S15). We initially measured the RIXS-AD with the magnetic field nearly parallel to $\boldsymbol{k}_{\boldsymbol{i n}}$ as a reference measurement. In this case the magnetic field is oriented along a high symmetry crystallographic direction and the angle between the linear incident polarization $\left(\boldsymbol{\epsilon}_{\boldsymbol{i n}}\right)$ and the magnetic field nearly does not change as a function of the sample rotation. These choices simplify the angular dependence and serve as a benchmark to analyze the RIXS spectra.

Although the experimental RIXS planes measured in the horizontal $\left(\phi=0^{\circ}\right)$ and vertical $\left(\phi=90^{\circ}\right)$ configurations show a broad single pre-edge peak (Fig. S9a and $\mathrm{b}$ ), it is possible to identify three main features in the experimental dichroism signal (Fig. 2b). Theoretical calculation of the RIXS dichroism signal shows that the quadrupolar contributions of the $\mathrm{Fe}^{3+} T_{d}$ and $O_{h}$ sites (a)

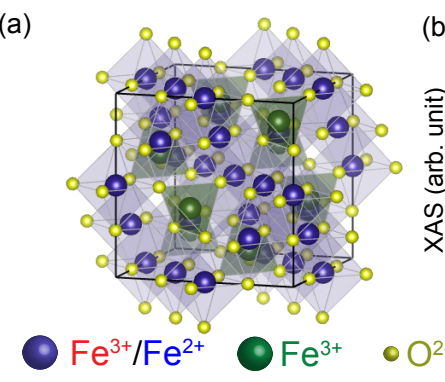

(b)
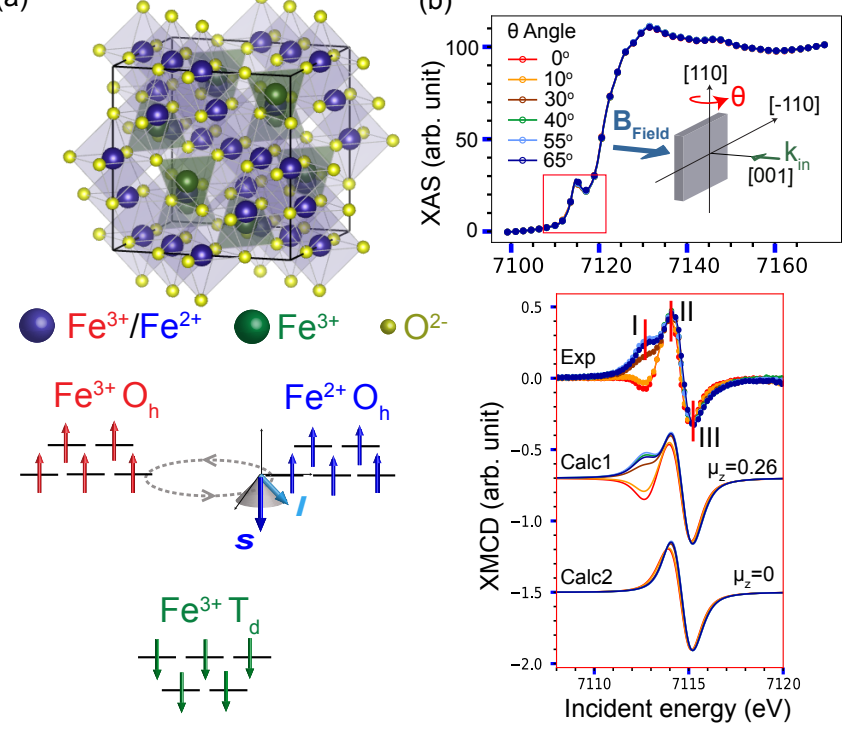

FIG. 1. (a) The cubic unit cell of $\mathrm{Fe}_{3} \mathrm{O}_{4}$ and the magnetic coupling between the Fe sites. Octahedral $\left(O_{h}\right) \mathrm{Fe}^{3+}$ and $\mathrm{Fe}^{2+}$ ions are antiferromagnetically coupled to the tetrahedral $\left(T_{d}\right) \mathrm{Fe}^{3+}$ ions. (b) $\mathrm{Fe} K$-edge measurements in $\mathrm{Fe}_{3} \mathrm{O}_{4}$ single crystal performed at Beamline ID12 of the European Synchrotron Facility (ESRF) [42]. The top panel shows XAS results as a function of the sample azimuthal angle $\theta$. The bottom panel shows the corresponding XMCD experimental (dotted) and theoretical (solid) Fe $K$ pre-edge signals. Two model calculations are presented: $i$ )-Calc 1 is the optimized calculation where a partially quenched orbital magnetic moment of $0.26 \mu_{\mathrm{B}}$ per unit formula of $\mathrm{Fe}_{3} \mathrm{O}_{4}$ was concluded, and ii)- Calc 2 is the theoretically expected XMCD signal for a fully quenched orbital magnetic moment scenario.

nearly cancel out (see Fig. S10) and hence the three features labelled in Fig. $2 \mathrm{~b}$ are dominantly attributed to the formal $\mathrm{Fe}^{2+}$ ions. This is consistent with the XMCD results, where we found that the angular dependence is only visible at $\mathrm{Fe}^{2+}$. The calculated RIXS dichroic plane of $\mathrm{Fe}^{2+}$ reproduces the three main spectral features. The calculation only misses a weak feature at incident energy $\sim 7115.1 \mathrm{eV}$ which is associated with the electric dipole transition at the $\mathrm{Fe}^{3+}$ A site due to onsite $3 d-4 p$ orbital mixing.

The full $360^{\circ}$ experimental (theoretical) angular dependence of the three main spectral features can be seen in Fig. 2c. The angular dependence is twofold and a $90^{\circ}$ angular shift is observed between the first feature and both the second and third features. A first explanation of the general angular dependence can be provided by analysing the $1 s^{1} 3 d^{7}$ intermediate states. Projections of the intermediate states associated with nonzero transition matrix elements onto cubic crystal field (i.e. $O_{h}$ ) configurations were calculated using the program CTM4DOC [43]. The first feature arises dominantly from excitations to the $t_{2 g}$ orbitals, that are $90^{\circ}$ 
angular shifted w.r.t. the second and third features corresponding to excitations dominantly into the $e_{g}$ orbitals [39]. This $90^{\circ}$ angular shift does not contradict the general angular behavior as formulated by Brouder [44]. The normalization as defined in Fig. 2 is responsible for the orientation of the lobes of the angular distribution: when the signal is positive, the lobes point towards $0^{\circ}$ and $180^{\circ}$ whereas they point towards $90^{\circ}$ and $270^{\circ}$ when the signal is negative. We note that the angular dependence is anisotropic where the intensity of the third feature at $\phi=180^{\circ}$ is smaller than that at $\phi=0^{\circ}$. This is related to the anisotropy in the detection in combination with a small offset angle $(\delta)$ of the magnetic field relative to the rotation axis i.e. the detector position relative to the sample [45-47]. In addition, our theoretical model assumes that the detection system is a single point while in reality four Ge (440) analyzer crystals were used. The minor discrepancy between the experiment and the calculations could be attributed to this facts.

We examined the coupling of the spin and orbital degrees of freedom by displacing the magnetic field $50^{\circ}$ from the high symmetry [001] direction (refer to Fig. 3a). The orientation of the magnetic field corresponds to the $\left[\frac{-\cos \left(40^{\circ}\right)}{\sqrt{2}}, \frac{\cos \left(40^{\circ}\right)}{\sqrt{2}}, \sin \left(40^{\circ}\right)\right]$ direction. Orienting the magnetic field in a low symmetry direction aligns the net spin magnetic moment parallel to the field. If the orbital magnetic moment is not fully quenched, it consequently re-aligns towards the low symmetry direction. The final orientation of the net magnetic moment depends on the strength of the competing interactions such as magnetic exchange, spin-orbit coupling and distortion. Hence, the angular shift of the maximum intensity of the excitations can be used to quantify magnetic-moment-induced distortion of the electron cloud. A careful analysis of the full $360^{\circ}$ angular dependence exhibits a peculiar $10^{\circ}$ angular shift of the maximum intensity between the second and third features in Fig. 3b.

Theoretical calculation of the angular dependences are presented in Fig. 3c. The model captures the essential aspects of the angular dependence and in particular the $10^{\circ}$ angular shift of the maximum intensity. The angular shift $(\Omega)$ quantified by fitting the angular dependence to a $\cos ^{2}(\phi+\Omega)$ function for the three features is reported in Tab. S4. The anisotropy of the angular dependence is not well reproduced, likely due to a small misalignment of the magnetic field that has not been included in the calculations (see Supplementary). This however does not affect our interpretation because the model captures the angular shift sufficiently well (Fig. S18) and can reproduce consistently all data including the RIXS-AD signal in the first configuration and the XMCD data. It is now important to highlight the key ingredients responsible for this angular shift. The first factor is the static trigonal distortion. The relative orientation of the exchange interaction with respect to the local trigonal distortion varies between the four sites leading to anisotropic effects and generates four non-equivalent Fe B sites. The theoretical RIXS-AD for the four sites are shown in Fig. S12. The second factor is the effect of dynamical distortion that produces two subclasses of the Fe B sites, namely, sites 1 and 2 forming one subclass and sites 3 and 4 forming the other. It is only when the dynamical distortion effect is taken into consideration can the experimental RIXS-AD angular shift with its magnetic contribution be reproduced (see Fig. S13). The RIXS-AD signal obtained in the absence of magnetization is reported in Fig.S14 and the influence of the direction or the orientation of the magnetization in Figs. S14 to S18. An energy shift of $0.2 \mathrm{eV}$ was found between the two subclasses.

We interpret the formation of these two subclasses as a result of a dynamical Jahn-Teller distortion at the $\mathrm{Fe}$ $\mathrm{B}$ sites. The magnitude of the static trigonal distortion lies close to the phonon energies of $\mathrm{Fe}_{3} \mathrm{O}_{4}[50,51]$, the magnetic exchange interaction and spin-orbit coupling, leading to a situation where electron-phonon interaction, dynamical Jahn-Teller and Kugel-Khomskii interactions all play a role in determining the low energy state. We treat this dynamical variation of the distortion in a first approximation as a small change in the bond lengths over the four sites giving rise to a small energy shift. This is a reasonable first approximation because the electronic structure adapts almost instantaneously to the crystallographic structure (i.e. the electronic motion is much faster than the nuclear motion). In this case, the effect of phonons could be simulated as a static distribution of bond lengths leading to a shift in energy between the four sites. This is an acceptable practice in XAS theory as demonstrated by Nemausat et. al. [52] where thermal fluctuations were shown to be well reproduced by averaging a series of static configurations. Although theoretical studies that treat simultaneously the electronic and the lattice degrees of freedom such as molecular dynamics and Monte Carlo simulations are required to comprehend the precise effect of the dynamical distortion, we point out that numerous theoretical works concluded the essential role of the strong electron-phonon coupling in the presence of strong electron correlations leading to dynamical Jahn-Teller distortion and the creation of polarons [53-56]. In particular, Piekarz et. al. [53, 54] identified the highly dispersive $X_{3}$ phonon mode as a primary order parameter of the Verwey transition which splits the four Fe B sites into two subclasses. This agrees rather well with our observation.

We have undergone the task of simulating various $\mathrm{X}$-ray spectroscopic measurements on the basis of our model. In particular, we focused on comparing $L_{3}$ XMCD $[2,7]$ and $L_{3}$ RIXS [7] measurements to our simulations. Our model can reproduce the experimental data and notably it captures the recently reported $L_{3}$ RIXS angular dependence well. The existence of this dynamical distortion is furthermore supported by various ex- 

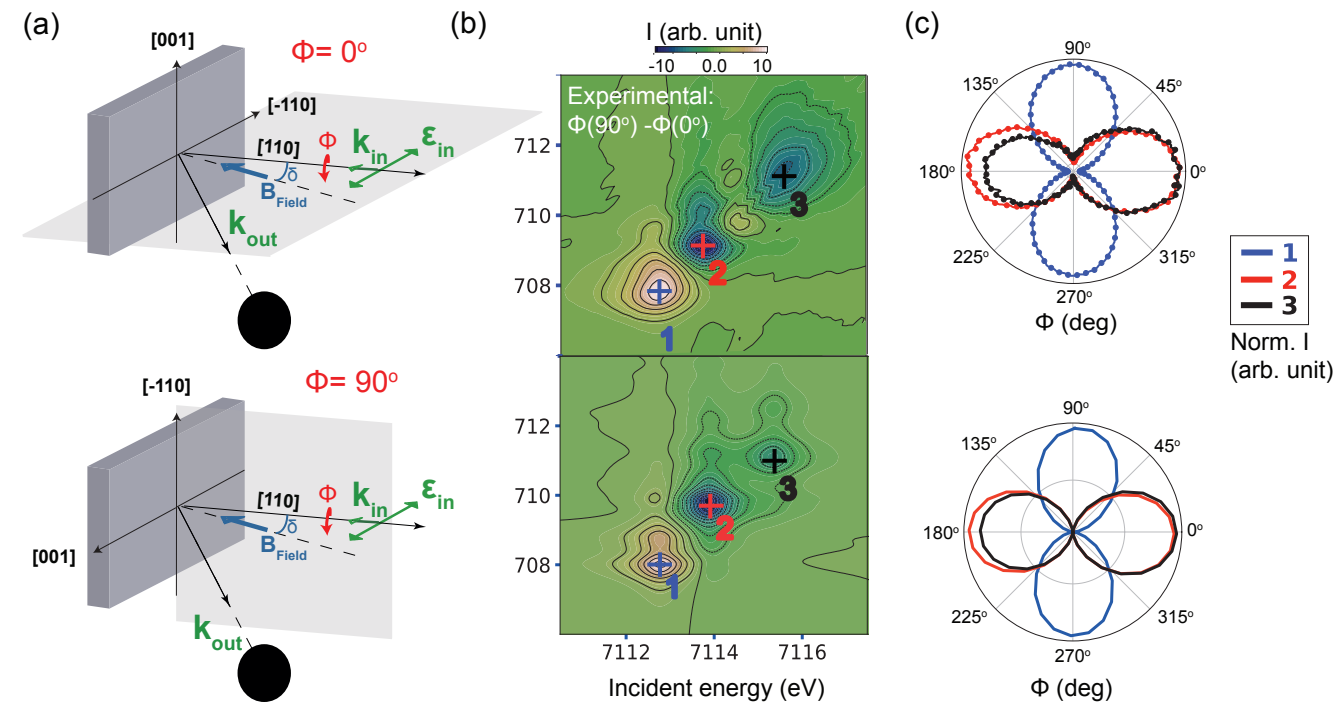

FIG. 2. Fe $1 s 2 p$ RIXS-AD measurements performed at Beamline ID26 of the ESRF [48, 49]. (a) A sketch of the scattering geometry employed. The magnetic field $\left(\boldsymbol{B}_{\boldsymbol{F i e l d}}\right)$ is aligned nearly parallel to the incident wave-vector $\left(\boldsymbol{k}_{\boldsymbol{i n}}\right)$ which corresponds to the [110] direction. (b) Experimental and theoretical dichroism RIXS planes computed as the difference between the RIXS plane at $\phi=90^{\circ}$ and at $\phi=0^{\circ}$. The full experimental (dotted) and theoretical (solid) $360^{\circ}$ RIXS-AD signals of the features labelled 1, 2 and 3 in the RIXS dichroic maps are shown in (c) respectively. The angular dependence signal is normalized as:

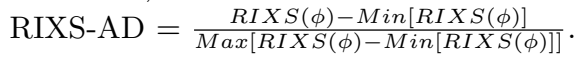
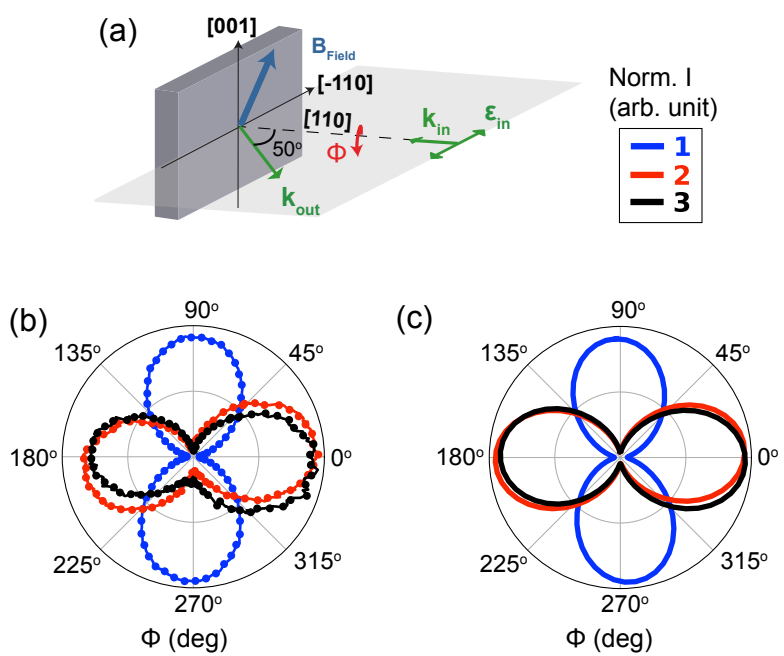

FIG. 3. Fe $1 s 2 p$ RIXS-AD measurements. (a) A sketch of the scattering geometry employed. The magnetic field $\left(\boldsymbol{B}_{\text {Field }}\right)$ is aligned to the $\left[\frac{-\cos \left(40^{\circ}\right)}{\sqrt{2}}, \frac{\cos \left(40^{\circ}\right)}{\sqrt{2}}, \sin \left(40^{\circ}\right)\right]$ direction. The angular dependence is measured by rotating the sample about the $[110]$ direction ( $\phi$ rotation). $\phi=0^{\circ}$ is defined when the incident polarization vector $\left(\boldsymbol{\epsilon}_{\boldsymbol{i n}}\right)$ is aligned to the $[-110] \mathrm{di}-$ rection. The experimental (dotted) and calculated (solid) angular dependence of the three main features (labeled 1, 2 and 3) are shown in panels (b) and (c).

perimental work such as diffuse scattering experiments using both neutrons [57] and X-rays [58], EXAFS [59], anomalous phonon broadening [60], and pump-probe Xray diffraction and optical reflectivity [61].
The presence of four non-equivalent $\mathrm{Fe} \mathrm{B}$ sites in the high temperature phase has rather interesting implications. Overall, we find that the average orbital magnetic moment deduced by XMCD and RIXS-AD is the same $\left(0.26 \pm 0.03 \mu_{\mathrm{B}}\right.$ per unit formula of $\mathrm{Fe}_{3} \mathrm{O}_{4}$ as illustrated in Fig. 1b and Fig. 4f), however the RIXS-AD measurement demonstrates that the average quantity is not sufficient to describe the orbital magnetic moment in $\mathrm{Fe}_{3} \mathrm{O}_{4}$. This is a result of the non-collinear orbital ordering arising from the interplay between trigonal distortion effects (static and dynamical), spin-orbit coupling and exchange interaction at the formal $\mathrm{Fe}^{2+}$ ions. The orbital magnetic moment per $\mathrm{Fe}^{2+}$ ion is predicted to have a strong dependence on the magnetic field in contrary to the spin magnetic moment which is collinear to the magnetic field. Fig. 4 illustrates the dependence of the orbital magnetic moments on the orientation of the magnetic field when we rotate it about the [110] direction for the four sites independently. Large non-collinear orbital contributions that tilt as much as $82^{\circ}$ away from the magnetic spin moment orientation are present. Furthermore, the collinear contribution per site ranges from 0 to $150 \%$ of the average quantity as a function of the orientation of the field. Remarkably, the average orbital magnetic moment for the four sites remains nearly constant (Fig. 4f).

The large discrepancies regarding the orbital magnetic moment of $\mathrm{Fe}$ in $\mathrm{Fe}_{3} \mathrm{O}_{4}$ can now be understood in light of the large non-collinear contribution, the site dependency and the magnetic field angular dependence. Experiments sensitive to the effective orbital magnetic moment yield 
(a)

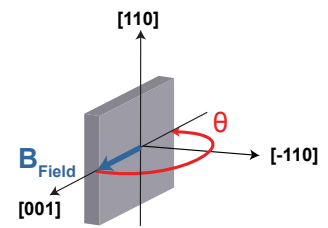

(b) Site 1

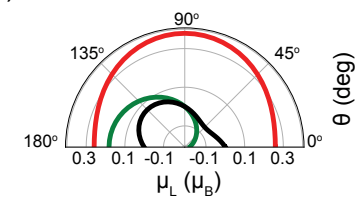

(d) Site 3

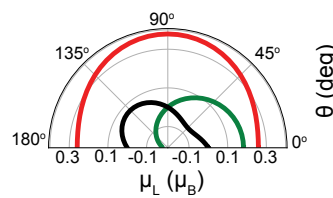

(c) Site 2

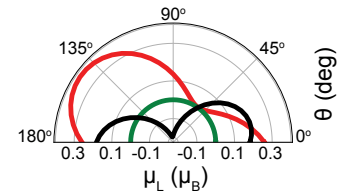

(e) Site 4

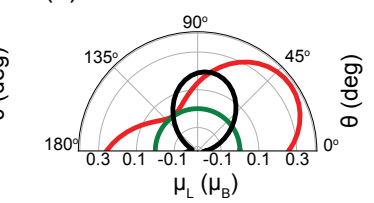

(f) Average
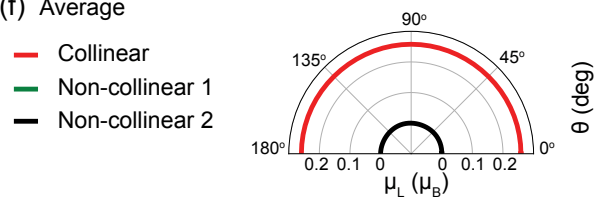

FIG. 4. The angular dependence of the orbital magnetic moment $\left(\mu_{L}\right)$ of the four $\mathrm{Fe}^{2+}$ ions as a function of the rotation of the magnetic field $\left(\boldsymbol{B}_{\text {Field }}\right)$ about the [110] orientation. (a) Sketch of the rotation geometry. The angular dependence of the orbital magnetic moment projected along the direction of $\boldsymbol{B}_{\text {Field }}$ and two perpendicular non-collinear contributions are shown in panel (b), (c), (d) and (e). The average orbital magnetic moment of the four $\mathrm{Fe}^{2+}$ ions are shown in (f). Note that the two non-collinear contributions in (f) overlap.

different results to those sensitive to the projected average quantity, or the average of the squared projected quantity. Moreover, variations as a function of the orientation of the magnetic field are expected for experiments sensitive to the non-averaged quantity. This ordering of the orbital magnetic moment is predicted to be short-ranged due to the dynamical distortions at the high temperature phase of $\mathrm{Fe}_{3} \mathrm{O}_{4}$. The combination of $1 s 2 p$ RIXS-AD and XMCD provides a powerful tool to quantify site-selectively non-collinear magnetic ordering with bulk sensitivity. Finally, we show that the orbital degree of freedom is an important precursor for the Verwey transition in $\mathrm{Fe}_{3} \mathrm{O}_{4}$ given the fact that it is coupled to a primary order parameter.

We acknowledge the staff of beamlines ID12 and ID26 of the European synchrotron radiation facility for their help in setting up and running the experiments. A. van der Eerden, S. Deelen, P. Theven and H. Vitoux are thanked for their efforts in building up the setup. We are grateful for P. Zimmerman and A. van der Eerden and A. Ismail for assisting during the synchrotron measurements at beamline ID26. We are grateful for the fruitful discussions with G. Subías, J. Garcia, J. Blasco and V. Vercamer. M. Delgado is thanked for providing and assisting us to use the program CTM4DOC. We thank A. Bosak and M. Hussein for their help with the $\mathrm{X}$-ray diffraction measurements and sample characterisation. Many thanks to R.-P. Wang for the discussions and suggestions. We acknowledge financial support from COST Action MP1306 (EUSpec). M. Sikora acknowledges support from National Science Center of Poland (2014/14/E/ST3/00026). This work was financed by the ERC advanced Grant XRAYonACTIVE No. 340279.

* H.M.E.A.Elnaggar@uu.nl

$\dagger$ F.M.F.deGroot@uu.nl

[1] E. Goering, M. Lafkioti, S. Gold, and G. Schütz, J. Magn. Magn. Mater. 310, e249 (2007).

[2] E. Goering, S. Gold, M. Lafkioti, and G. Schütz, Europhys. Lett. 73, 97 (2006).

[3] D. J. Huang, C. F. Chang, H.-T. Jeng, G. Y. Guo, H.-J. Lin, W. B. Wu, H. C. Ku, A. Fujimori, Y. Takahashi, and C. T. Chen, Phys. Rev. Lett. 93, 077204 (2004).

[4] E. Goering, Phys. Status Solidi B 248, 2345 (2011).

[5] E. Arenholz, G. van der Laan, R. V. Chopdekar, and Y. Suzuki, Phys. Rev. B 74, 094407 (2006).

[6] H. Y. Huang, Z. Y. Chen, R. P. Wang, F. M. De Groot, W. B. Wu, J. Okamoto, A. Chainani, J. S. Zhou, H. T. Jeng, G. Y. Guo, et al., Nat. Commun. 8, 15929 (2017).

[7] H. Elnaggar, R. P. Wang, S. Lafuerza, E. Paris, A. C. Komerak, H. Guo, Y. Tseng, D. Mcnelly, F. Frati, M. W. Haverkort, et al., arXiv:1811.04836 [cond-mat.strel] (2018).

[8] Y. Li, P. A. Montano, B. Barbiellini, P. E. Mijnarends, S. Kaprzyk, and A. Bansil, J. Phys. Chem. Solids 68, 1556 (2007).

[9] J. A. Duffy, J. W. Taylor, S. B. Dugdale, C. ShentonTaylor, M. W. Butchers, S. R. Giblin, M. J. Cooper, Y. Sakurai, and M. Itou, Phys. Rev. B 81, 134424 (2010).

[10] I. Leonov, A. N. Yaresko, V. N. Antonov, M. A. Korotin, and V. I. Anisimov, Phys. Rev. Lett. 93, 146404 (2004).

[11] M. Coey, Nature 430, 155EP (2004).

[12] Y. Tokura and N. Nagaosa, Science 288, 462 (2000).

[13] P. G. Radaelli, New J. Phys. 7, 53 (2005).

[14] J. Schlappa, C. Schüßler-Langeheine, C. F. Chang, H. Ott, A. Tanaka, Z. Hu, M. W. Haverkort, E. Schierle, E. Weschke, G. Kaindl, et al., Phys. Rev. Lett. 100, 026406 (2008).

[15] S. B. Wilkins, S. Di Matteo, T. A. W. Beale, Y. Joly, C. Mazzoli, P. D. Hatton, P. Bencok, F. Yakhou, and V. A. M. Brabers, Phys. Rev. B 79, 201102 (2009).

[16] A. Tanaka, C. F. Chang, M. Buchholz, C. Trabant, E. Schierle, J. Schlappa, D. Schmitz, H. Ott, P. Metcalf, L. H. Tjeng, et al., Phys. Rev. Lett. 108, 227203 (2012).

[17] A. Tanaka, C. F. Chang, M. Buchholz, C. Trabant, E. Schierle, J. Schlappa, D. Schmitz, H. Ott, P. Metcalf, L. H. Tjeng, et al., Phys. Rev. B 88, 195110 (2013).

[18] D. Dunlop and O. Özdemir, Rock magnetism (Cambridge Univ. Press., 1997).

[19] E. J. W. Verwey, Nature 144, 327 (1939). 
[20] N. F. Mott, Rev. Mod. Phys. 40, 677 (1968).

[21] M. Imada, A. Fujimori, and Y. Tokura, Rev. Mod. Phys. 70, 1039 (1998).

[22] M. S. Senn, J. P. Wright, and J. P. Attfield, Nature 481, 173 (2012).

[23] P. Carra, B. T. Thole, M. Altarelli, and X. Wang, Phys. Rev. Lett. 70, 694 (1993).

[24] J. P. Wright, A. M. T. Bell, and J. P. Attfield, Solid State Sci. 2, 747 (2000).

[25] F. Walz, J. Phys. Condens. Matter 14, R285 (2002).

[26] S. Klotz, G. Steinle-Neumann, T. Strässle, J. Philippe, T. Hansen, and M. J. Wenzel, Phys. Rev. B 77, 012411 (2008).

[27] P. Novák and V. Chlan, Phys. Rev. B 81, 174412 (2010).

[28] D. Levy, R. Giustetto, and A. Hoser, Phys. Chem. Minerals 39, 169 (2012).

[29] D. I. Khomskii and M. V. Mostovoy, J. Phys. A: Math. Gen. 36, 9197 (2003).

[30] P. Kuiper, B. Searle, L.-C. Duda, R. Wolf, and P. van der Zaag, J. Electron Spectros. Relat. Phenomena. 86, 107 (1997).

[31] R. A. D. Pattrick, G. van der Laan, C. M. B. Henderson, P. Kuiper, E. Dudzik, and D. J. Vaughan, Eur. J. Mineral. 14, 1095 (2002).

[32] C. Carvallo, P. Sainctavit, M. A. Arrio, N. Menguy, Y. Wang, G. Ona-Nguema, and S. Brice-Profeta, Am. Mineral. 93, 880 (2008).

[33] X. Zhu, S. S. Kalirai, A. P. Hitchcock, and D. A. Bazylinski, J. Electron Spectros. Relat. Phenomena 199, 19 (2015).

[34] B. Liu, C. Piamonteze, M. U. Delgado-Jaime, R.-P. Wang, J. Heidler, J. Dreiser, R. Chopdekar, F. Nolting, and F. M. F. de Groot, Phys. Rev. B 96, 054446 (2017).

[35] H. Uzu and A. Tanaka, J. Phys. Soc. Jpn. 77, 074711 (2008).

[36] M. W. Haverkort, M. Zwierzycki, and O. K. Andersen, Phys. Rev. B 85, 165113 (2012).

[37] Y. Lu, M. Höppner, O. Gunnarsson, and M. W. Haverkort, Phys. Rev. B 90, 085102 (2014).

[38] M. W. Haverkort, G. Sangiovanni, P. Hansmann, A. Toschi, Y. Lu, and S. Macke, EPL 108, 57004 (2014).

[39] T. E. Westre, P. Kennepohl, J. G. DeWitt, B. Hedman, K. O. Hodgson, and E. I. Solomon, J. Am. Chem. Soc. 119, 6297 (1997).

[40] M.-A. Arrio, S. Rossano, C. Brouder, L. Galoisy, and G. Calas, EPL 51, 454 (2000).

[41] V. Vercamer, M. O. J. Y. Hunault, G. Lelong, M. W. Haverkort, G. Calas, Y. Arai, H. Hijiya, L. Paulatto, C. Brouder, M.-A. Arrio, et al., Phys. Rev. B 94, 245115 (2016).
[42] A. Rogalev and F. Wilhelm, Phys. Met. Metallogr. 116, 1285 (2015).

[43] M. U. Delgado-Jaime, K. Zhang, J. Vura-Weis, and F. M. F. De Groot, J. Synchrotron Radiat. 23, 1264 (2016).

[44] C. Brouder, J. Phys. Condens. Matter 2, 701 (1990).

[45] See Supplemental Material for details about the anisotropic behaviour which includes Refs. [46,47].

[46] C. Roth, F. U. Hillebrecht, H. Rose, and E. Kisker, Phys. Rev. Lett. 70, 3479 (1993).

[47] A. Juhin, C. Brouder, and F. M. F. de Groot, Open Phys. 12, 323 (2014).

[48] C. Gauthier, V. A. Solé, R. Signorato, J. Goulon, and E. Moguiline, J. Synchrotron Rad. 6, 164 (1999).

[49] P. Glatzel, T. C. Weng, K. Kvashnina, J. Swarbrick, M. Sikora, E. Gallo, N. Smolentsev, and R. A. Mori, J. Electron Spectros. Relat. Phenomena 188, 17 (2013).

[50] L. V. Gasparov, D. B. Tanner, D. B. Romero, H. Berger, G. Margaritondo, and L. Forró, Phys. Rev. B 62, 7939 (2000).

[51] B. Handke, A. Kozlowski, K. Parlinnski, J. Przewoznik, T. Slezak, A. I. Chumakov, L. Niesen, Z. Kakol, and J. Korecki, Phys. Rev. B 71, 144301 (2005).

[52] R. Nemausat, D. Cabaret, C. Gervais, C. Brouder, N. Trcera, A. Bordage, I. Errea, and F. Mauri, Phys. Rev. B 92, 144310 (2015).

[53] P. Piekarz, K. Parlinski, and A. M. Oles, Phys. Rev. Lett. 97, 156402 (2006).

[54] P. Piekarz, K. Parlinski, and A. M. Oles, Phys. Rev. B 76, 165124 (2007).

[55] S. Borroni, G. S. Tucker, F. Pennacchio, J. Rajeswari, U. Stuhr, A. Pisoni, J. Lorenzana, H. M. RØnnow, and F. Carbone, New J. Phys. 19, 103013 (2017).

[56] J. Cumby and J. P. Attfield, Nat. Commun. 8, 14235 EP (2017).

[57] Y. Yamada, N. Wakabayashi, and R. M. Nicklow, Phys. Rev. B 21, 4642 (1980).

[58] A. Bosak, D. Chernyshov, M. Hoesch, P. Piekarz, M. Le Tacon, M. Krisch, A. Kozłowski, A. M. Oleś, and K. Parlinski, Phys. Rev. X 4, 011040 (2014).

[59] G. Subías, J. García, and J. Blasco, Phys. Rev. B 71, 155103 (2005).

[60] M. Hoesch, P. Piekarz, A. Bosak, M. Le Tacon, M. Krisch, A. Kozłowski, A. M. Oleś, and K. Parlinski, Phys. Rev. Lett. 110, 207204 (2013).

[61] S. de Jong, R. Kukreja, C. Trabant, N. Pontius, C. F. Chang, T. Kachel, M. Beye, F. Sorgenfrei, C. H. Back, B. Bräuer, et al., Nat. Mater. 12, 882EP (2013). 